\title{
Tendencias y perspectivas del marketing en las pymes
}

\section{Juan Carlos Suárez Pérez, Osmany Pérez Barral}

Pontificia Universidad Católica del Ecuador Sede Ambato

\author{
Escuela de Administración de Empresas, Pontificia Universidad Católica del Ecuador Sede Ambato
}

La investigación comprende y analiza la situación actual de las estrategias de marketing en las pequeñas y medianas empresas (pymes), pues se ha evidenciado que están diseñadas de manera empírica, es decir, sin un sustento técnico que garantice el éxito de la planificación para su desarrollo y sostenibilidad. Asimismo, el estudio profundiza en la realidad de estas organizaciones, y en las tendencias y perspectivas del marketing que se ajusten a sus necesidades para hacerlas más competitivas y se posicionen en el mercado. En este sentido, el objetivo de este artículo es analizar las tendencias y perspectivas del marketing en las pymes de la ciudad de Ambato. Para ello, se aplicó una metodología cualitativa, que permitió contextualizar e indagar información natural, holística y flexible. Los resultados se enmarcan en la determinación de la planificación estratégica de las pymes en la ciudad que fue el objeto de estudio. Se tuvo la finalidad de mejorar el funcionamiento, consolidar su marca y lograr digitalizar en el futuro los negocios más allá del tamańo que tengan. Todo esto contribuye a su propio posicionamiento mediante la optimización de los recursos, la mejora de la tecnología, y la posibilidad de aplicar estrategias más eficientes que las hagan crecer y expandirse.

Palabras clave: marketing, marketing digital, marketing en pymes, tendencias y perspectivas de marketing

\section{Marketing trends and perspectives in SMEs}

The research understands and analyzes the current situation of marketing strategies in Small and medium-sized enterprises (SMEs), since it has been shown that they are designed empirically, that is, without a technical support that guarantees the success of planning for their development and sustainability. Likewise, the study delves into the reality of these organizations, and the marketing trends and perspectives that fit their needs to make them more competitive and position themselves in the market. In this sense, the objective is to analyze the trends and perspectives of marketing in SMEs in the city of Ambato, for this, a qualitative methodology was applied, which allowed contextualizing and investigating natural, holistic and flexible information. The results are part of the determination of the strategic planning of SMEs in the city under study; in order to improve its operation, consolidate its brand and digitize businesses in the future, no matter how small they may be, since this contributes to their own positioning, through the optimization of resources, the improvement of technology and the possibility of applying more efficient strategies that make them grow and expand.

Keywords: marketing, digital marketing, marketing in SMEs, marketing trends and perspectives 


\section{Tendências e perspectivas de marketing nas PMEs}

A pesquisa compreende e analisa a situação atual das estratégias de marketing nas Pequenas e Médias Empresas (PMEs), uma vez que se tem demonstrado que são concebidas empiricamente, ou seja, sem um suporte técnico que garanta o sucesso do planejamento para o seu desenvolvimento e sustentabilidade. Da mesma forma, o estudo investiga a realidade dessas organizaçóes, as tendências e perspectivas de marketing que se enquadram nas suas necessidades para torná-las mais competitivas e posicionar-se no mercado. Nesse sentido, o objetivo é analisar as tendências e perspectivas do marketing nas PMEs da cidade de Ambato, para isso, foi aplicada uma metodologia qualitativa, que permitiu contextualizar e investigar informaçôes naturais, holísticas e flexíveis. Os resultados fazem parte da determinação do planejamento estratégico das PMEs da cidade em estudo; Para melhorar o seu funcionamento, consolidar a sua marca e digitalizar os negócios do futuro, por menores que sejam, pois isso contribui para o seu próprio posicionamento, através da otimizaçáo de recursos, do aprimoramento da tecnologia e da possibilidade de aplicação mais eficiente estratégias que os fazem crescer e se expandir.

Palavras-chave: marketing, marketing digital, marketing em PMEs, tendências e perspectivas de marketing

\section{Introducción}

Según el Instituto Nacional de Estadística y Censos (INEC, 2019), en Ambato, las pequeñas y medianas empresas (en adelante, pymes) constituyen el 93,53\% de las unidades productivas de la ciudad. En ese sentido, se tratan del motor de la economía local, por lo que requieren de estrategias para gestionar eficientemente sus recursos, ser más competitivas y consolidarse en el mercado. Al respecto, la presente investigación se centra en el marketing, dado que la globalización y los avances tecnológicos permiten que los consumidores estén más informados y tiendan a ser más exigentes. Por ello, las empresas deben innovar en su planificación estratégica para adaptarla a la nueva era de la información y basarla en el buyer persona.

Pese a la importancia del marketing, las cifras no son alentadoras en Ecuador, pues, de acuerdo con Oller y Játiva (2016), solo el 61,7\% de empresas tiene nociones de qué trata la mercadotecnia, el 52,1\% la concibe únicamente como publicidad y presencia en internet (redes sociales), el 36,2\% no tiene clara su importancia y/o no cuentan con los recursos necesarios, y solo el 9,6\% la ve como un área estratégica. A su vez, Lovato, López y Acosta (2018) indican que las pymes nacionales tienen un alto nivel de empirismo en todos sus procesos, debido a la falta de inversión en capacitación e innovación. En síntesis, se manejan estrategias sin el sustento técnico que garantice su éxito.

Frente a esa realidad, en su desarrollo dentro del mercado, las pymes se han visto limitadas por grandes organizaciones que tienen un mejor posicionamiento y mejores condiciones económicas, por lo que las superan tecnológicamente y en niveles de ventas. De esta manera, las pymes se ven afectadas en su crecimiento al tener que competir con las grandes empresas, pues no cuentan con estructuras sólidas que les permitan ser sostenibles en el tiempo y en el mercado. Así, se evidencia que, en Ecuador, este tipo de organización no ha aprovechado la masificación del internet y las plataformas digitales para generar nuevos clientes. Dada esta situación, se plantea el siguiente problema científico: ¿cómo se puede mejorar el posicionamiento de las pequeñas y medianas empresas en la ciudad de Ambato? 
El objetivo de la investigación es analizar las tendencias y perspectivas del marketing en las pymes de la ciudad de Ambato. Para ello, se fundamentaron teóricamente las variables que son objeto de estudio, es decir, las tendencias y perspectivas del marketing en las pymes, con la finalidad de indagar su evolución como elemento de gestión y crecimiento a través del tiempo. Además, se realizó un diagnóstico de la situación actual de este tipo de empresas y sus estrategias de marketing. Por último, se desarrolló un análisis de las perspectivas en el mercado.

\section{Evolución de las tendencias y perspectivas del marketing en las pymes}

Los orígenes del marketing tienen como punto de partida la creación de la imprenta por parte de Gutenberg en el año 1450. En un inicio, autores como E.D Jones, Fred E. Clarck y Philip Kotler definieron al "marketing» como aquellos esfuerzos mercadológicos que se centran en el producto. Esto originó lo que se conoce como "marketing 1.0», que se caracterizaba por ser unidireccional, debido a que la televisión, la radio y la prensa no permitían los procesos idóneos de retroalimentación de los resultados de una campaña de mercadotécnica. En este sentido, Rodríguez, Pineda, y Castro (2020) consideran que la evolución $\mathrm{y}$ las tendencias del marketing provienen de una mutación de lo tradicional. Asimismo, Fuciu y Dumitrescu (2018) mencionan que el principal cambio que originó el marketing 2.0 fue el paso de lo exclusivo a lo inclusivo, que supuso que el consumidor se convierta en el centro de las estrategias mercadológicas. En esta etapa, se incluyen a los medios interactivos para promover la conexión de las empresas con sus clientes, con el propósito de obtener el feedback de los resultados y proponer mejoras en las campańas de marketing digital.
Posteriormente, las estrategias mercadológicas se enfocaron en los valores y en el interés por contribuir a la sociedad; así, se introdujo el término "marketing 3.0», que se ha caracterizado por ser multidireccional, dado el uso de medios interactivos. Finalmente, con el propósito social de mejorar el mundo tecnológico y humanizar las marcas de las organizaciones, el desarrollo de la tecnología digital condujo a la aparición del marketing 4.0, que es la tendencia más actual y global, pues sus características funcionales han acelerado los cambios en la vida de las personas y de las empresas.

Dado lo expuesto, se considera que la tendencia actual es la del marketing 4.0, que, según Kotler, Kartajaya, y Setiawan (2018), consiste en la profundización y ampliación de la mercadotecnia enfocada en el ser humano para satisfacer sus necesidades. Adicionalmente, Suárez (2018) indica que implica la predicción y anticipación a lo que va a suceder en el mercado, con la finalidad de identificar tendencias. Para ello, se apoya en el big data y la inteligencia de negocios. Estos aspectos deben ser considerados por las pymes para su crecimiento, pues permanecen gran cantidad de tiempo en el mercado con un criterio limitado y conformista de evolución y expansión.

A su vez, para Gonzáles (2017), Viteri, Herrera, y Basurto (2017), y Wereda y Woźniak (2019) la tendencia del marketing es el reflejo de la evolución de los clientes, ya que, en la era digital, están más informados, son más exigentes y buscan mejores experiencias al tomar decisiones de compra. Estos autores coinciden en que la mercadotécnica está sujeta a los avances tecnológicos, pues han sido los precursores de nuevas tendencias, como e-commerce, endomarketing, marketing sensorial, marketing experiencial, marketing de contenidos, big data, business intelligence y redes sociales, entre otras. Concretamente, las tendencias más relevantes corresponden a big data o manejo de 
grandes bases de datos a gran velocidad y con sustentos cuantificables (Goyzueta, 2015); marketing sensorial y experiencial, que, de acuerdo con Saboga y Rojas (2020), y Ortegón y Gómez (2016), se apoyan en los sentimientos para atraer clientes; e-commerce o transacciones comerciales que se realizan en la web (Mieles, Albán, Valdospin, \& Vera, 2018); marketing de contenidos, con el que se busca empoderar a los clientes (Pažèraitė \& Repovienè, 2018); y social media marketing, que, a través de las redes sociales, posibilita el desarrollo de estrategias de menor costo y mayor impacto (Real, Leyva, \& Heredia, 2014).

Las tendencias comentadas (big data, e-commerce, content marketing, y las redes sociales, entre otras) deben ser consideradas por las pymes en el futuro para poder adaptarse, y lograr un mayor desarrollo tecnológico y digital. De lo contrario, no podrán ser competitivas en un mercado tan hostil. En este sentido, de acuerdo a Alarcón y Granda (2018) las pymes deben innovar su planificación de marketing para mantenerse en el mercado, así como lo hacen las grandes empresas. Este criterio se materializa según los aportes de Hernández, López, y Salcedo (2019), que, en su estudio, corroboran que el acceso a la información, a las nuevas tecnologías y a la creatividad ha reducido la brecha entre las pymes y las grandes empresas. Los autores concluyen que eso permite que las pymes puedan ser más competitivas en el ámbito de la mercadotecnia; así, dichas organizaciones compiten en mejores o iguales condiciones que las existentes en la actualidad.

Asimismo, en esta investigación, se coincide con Cafaro (2019), quien expone que la digitalización de los negocios no es opcional, es decir, todas las empresas deben reinventarse e implementar herramientas digitales de marketing. Por otra parte, Striedinger (2018) plantea que existen limitantes para digitalizar, como, por ejemplo, la informalidad de los procesos, la existencia de polivalencia y multifuncionalidad de los propietarios, y el manejo emocional y empírico de los negocios. A partir de estas circunstancias, los autores plantean que la tendencia a adoptar debe ser el marketing 4.0, pues, más allá del tamaño, toda organización debe aplicar estrategias mercadológicas avanzadas, y asociadas a la informatización y al componente digital.

Con respecto al marketing 4.0, el e-commerce ha sido la tendencia de mayor acogida en el año 2020 a nivel local, pues los consumidores son más móviles y agnósticos de canal (Kotler et al., 2018). Asimismo, existe inclinación por las empresas que logren deslocalizar los puntos de venta, garanticen la flexibilidad de pago, generen nuevas relaciones, reduzcan costo y faciliten el acceso a mercado globales (León, Garzón, Meza, \& Macias, 2018): son factores que inciden en los clientes y su identificación con las organizaciones que aplican estas herramientas de gestión de marketing, pues les ahorran tiempo y movilidad.

Por otra parte, Enríquez (2019), Taylor (2019), Olivier (2020), y el portal especializado de Hubspot \& Talwalker (2020) consideran que las redes sociales son la base de la estrategia de marketing, pues ofrecen diversidad de herramientas y opciones que pueden ser aprovechadas por todas las empresas, incluidas las pymes. A nivel interno de la empresa, se mencionan también los administradores de anuncios, las estadísticas y los informes; a nivel externo (es decir, lo que ve el consumidor), se permiten viralizar contenidos, crear comunidades e interactuar directamente con los clientes, entre otras opciones menos costosas.

Además, existen otras tendencias que son aceptadas por los clientes, pues se apoyan en las redes sociales. Entre ellas, se encuentran los siguientes ejemplos: marketing de influencers, storytelling, chat bots, remixes en Instagram reels o TikTok, y gamming, entre otras. 
Todas estas estrategias bidireccionales del marketing contribuyen a conectar a la empresa con sus clientes, con el fin de mejorar la experiencia de estos y los empresarios, incrementar la fidelización, y disminuir el presupuesto en comparación a las otras estrategias mercadológicas tradicionales. Estas ideas se ven confirmadas por los criterios de Real et al. (2014), y Hoyos y Sastoque (2020) en sus estudios sobre la panificación del marketing. Tales autores señalan que, para garantizar el éxito de una planificación de marketing, no se pueden descuidar temas de la mercadotecnia tradicional, como la fuerza de venta, las políticas y valores empresariales, los canales de distribución, la investigación de mercado, la publicidad convencional, y la imagen corporativa, entre otros. Dichos aspectos son la base de la estrategia digital que abarca todas las tendencias mencionadas hasta este momento. Con ello, se ratifica que el éxito del marketing depende de las estrategias online y offline.

Finalmente, la globalización y la dinámica cambiante del mercado implican que las pymes deban pasar a la era de la digitalización de sus negocios. Para ello, requieren de creatividad y el uso de las distintas plataformas digitales, que, además, son menos costosas que los medios tradicionales de comunicación. Con tales innovaciones, se puede viralizar el contenido de marketing, llegar a mercados más amplios o mejor segmentados (dependiendo de los objetivos de marketing que se planteen); crear una estrategia digital sin el sustento técnico de las estrategias tradicionales, pero con adecuada segmentación de mercado (investigación previa); o crear estrategias de posicionamiento.

\section{Metodología}

La investigación es de tipo descriptiva-explicativa, diseño que, según Hernández Sampieri, Fernández y Baptista (2014), permite indagar y especificar las características y propiedades más relevantes de un fenómeno determinado. En este caso, se investigarán las tendencias y las perspectivas de marketing en las pymes, y cómo estas han evolucionado mediante los avances tecnológicos. Entonces, la investigación descriptiva permite mostrar con precisión qué tendencias de mercadotecnia son las más usuales hoy en día, y cómo contribuyen al posicionamiento de las empresas. El enfoque de la investigación es cualitativo, pues, tal como seńalan Hernández Sampieri et al. (2014), permite profundizar, contextualizar e indagar la información natural, holística y flexible que ha sido abordada en este trabajo.

La población que ha sido objeto de estudio está conformada por pymes de la provincia de Tungurahua, en donde, según el INEC (2019), existen 42.169 pymes. En ese sentido, se tomaron en consideración únicamente aquellas empresas relacionadas con servicios $(44,55 \%)$, comercio $(33,90 \%)$ y manufactura $(8,41 \%)$; así, se obtuvo una población de 36.628 pymes. Para la selección de la muestra, se procedió al cálculo con la siguiente formula: la probabilidad de éxito $(\mathrm{P})$ fue 0,50 , y la probabilidad de fracaso $(\mathrm{Q})$ fue 0,50; el nivel de confianza (Z) fue de $95 \%$, es decir, 1,76; y se tuvo como margen de error (e) el $5 \%$. Con esos valores, se procedió a calcular la muestra con la siguiente fórmula:

$$
\begin{gathered}
n=\frac{Z^{2} P Q N}{N e^{2}+Z^{2} P Q} \\
n=\frac{1.96^{2}(36,628)(0.50)(0.50)}{36,628(0.05)^{2}+1.96^{2}(0.50)(0.50)} \\
n=380
\end{gathered}
$$

Se obtuvo una muestra de 380 pymes, a las que se les aplicó una encuesta para conocer la situación actual de 
sus estrategias de marketing. La encuesta fue enviada a través de un formulario de Microsoft Forms a la totalidad (380) de empresas de la muestra. Sin embargo, únicamente respondieron 127 , es decir, $33 \%$ de la totalidad de la muestra. Aquellas empresas que no respondieron argumentaron falta de tiempo para proporcionar la información y otras simplemente no dieron razón. Posteriormente, se realizó un estudio comparativo de las ventajas y desventajas de las tendencias a través del tiempo de las estrategias de marketing, las cuales se resumen en la tabla 1 .

\section{Resultados}

La encuesta se aplicó a 127 pymes. De ellas, el 54\% son empresas de servicios; el 35\%, de comercio; y el $11 \%$, de manufactura. El tiempo promedio de años que tienen en el mercado es de 12 ańos y la media de número de trabajadores es de 13 personas. Como se observa en la tabla 1, las ventajas de las herramientas de marketing se enfocan fundamentalmente en que se puede lograr que exista un crecimiento de la oferta a partir del incremento de nuevos competido-

\section{Tabla 1. Ventajas y desventajas de las herramientas de marketing}

\begin{tabular}{|c|c|c|c|}
\hline Autores & Tendencias & Ventaja & Desventaja \\
\hline $\begin{array}{l}\text { Rivera (2015); } \\
\text { Ruizalba, Vallespín y } \\
\text { Pérez (2015); Suárez } \\
\text { (2018); Alin (2019); } \\
\text { Mendivelso y Lobos } \\
\text { (2019). }\end{array}$ & $\begin{array}{l}\text { Marketing } \\
1.0\end{array}$ & $\begin{array}{l}\text { Desarrolló la ventaja competitiva de las empresas que } \\
\text { aplicaban estrategias. } \\
\text { Desarrolló la oferta, es decir, aparecieron diversos } \\
\text { competidores con nuevos productos para atender las } \\
\text { necesidades de los consumidores. } \\
\text { A través de la investigación, permitió sistematizar } \\
\text { métodos y el conocimiento con el fin de alcanzar los } \\
\text { propósitos empresariales. }\end{array}$ & $\begin{array}{l}\text { No existían criterios de consumo, es decir, } \\
\text { se consumía lo que ofertaba el mercado sin } \\
\text { importar las características del producto. } \\
\text { Implica que la comunicación sea unidirec- } \\
\text { cional, es decir, no se escucha al cliente. } \\
\text { Se pierde la vista global del panorama, es } \\
\text { decir, las empresas no reconocían el mer- } \\
\text { cado en el que competían. } \\
\text { Implicar tener consumidores pasivos. }\end{array}$ \\
\hline $\begin{array}{l}\text { Erragcha y Romd- } \\
\text { hane (2014); Rivera } \\
\text { (2015); Suárez } \\
\text { (2018); Camacho } \\
\text { (2019). }\end{array}$ & $\begin{array}{l}\text { Marketing } \\
2.0\end{array}$ & $\begin{array}{l}\text { Se centra en el cliente, es decir, en satisfacer sus nece- } \\
\text { sidades y deseos. } \\
\text { La rentabilidad armoniza con la responsabilidad cor- } \\
\text { porativa. } \\
\text { Dinamizó la comunicación. } \\
\text { Adaptó las estrategias a la web } 2.0 \text {. }\end{array}$ & $\begin{array}{l}\text { La información se encuentra desorgani- } \\
\text { zada y redundante, por lo que es poco útil } \\
\text { según la necesidad de los clientes. } \\
\text { Muestra poca madurez de la inteligencia } \\
\text { artificial (en adelante, IA). }\end{array}$ \\
\hline $\begin{array}{l}\text { Rivera (2015); } \\
\text { Warrink (2015); } \\
\text { Camacho (2019); } \\
\text { Alin (2019); And- } \\
\text { hyka (2020). }\end{array}$ & $\begin{array}{l}\text { Marketing } \\
3.0\end{array}$ & $\begin{array}{l}\text { Concibe la inteligencia, el corazón y el espíritu de los } \\
\text { seres humanos, y no los ve como simples consumi- } \\
\text { dores. } \\
\text { Permite la masificación de los medios de comunica- } \\
\text { ción y el comercio electrónico. } \\
\text { Contribuye con la facilidad para viralizar los mensajes } \\
\text { e información de las empresas. }\end{array}$ & $\begin{array}{l}\text { Pese a que las estrategias estaban enfoca- } \\
\text { das en esta tendencia, la realidad no cum- } \\
\text { plía con su premisa de «hacer un mundo } \\
\text { mejor». } \\
\text { Sobredimensiona y/o exagera las estrategias } \\
\text { con el fin de que sean más competitivas. }\end{array}$ \\
\hline $\begin{array}{l}\text { Rodriguez, } \\
\text { Camargo, González } \\
\text { y Gorrin (2015); } \\
\text { Alin (2019); Pérez } \\
(2020) \text {. }\end{array}$ & $\begin{array}{l}\text { Marketing } \\
4.0\end{array}$ & $\begin{array}{l}\text { Predice el comportamiento de los consumidores para } \\
\text { satisfacerlo a través de herramientas tecnológicas. } \\
\text { Permite el monitoreo de las estrategias en tiempo real. } \\
\text { Permite la interacción de estrategias online y offline } \\
\text { para mayor competitividad. } \\
\text { Potencializa la creatividad de las empresas. }\end{array}$ & $\begin{array}{l}\text { Puede demandar cambios drásticos según } \\
\text { la evolución de la tecnología y el compor- } \\
\text { tamiento de los consumidores. } \\
\text { Genera adicción a las plataformas digitales. }\end{array}$ \\
\hline
\end{tabular}


res que ofrecen nuevos servicios. Esto coadyuva a que los clientes tengan mayores opciones de compras y los negocios puedan mejorar sus resultados económicosfinancieros, con el propósito de convertirse en negocios más consolidados que puedan extenderse a nivel nacional en un primer orden. A la vez, esto exige que la atención al cliente mejore para que se pueda alcanzar una alta fidelización de estos.

De la misma manera, los empresarios deben trabajar sobre las principales desventajas que se aprecian según el criterio de diversos autores. Por ejemplo, debe hacerse un estudio del tipo de consumo que puede generarse a partir de las expectativas y necesidades de los clientes, y la atención personalizada a estos; a partir de ello, se puede mejorar la tecnología de las organizaciones para garantizar productos y servicios de calidad. Para ello, se tiene que trabajar en el sistema informativo sobre el mercado y los clientes, pues, con ello, se garantiza un acercamiento entre clientes y negocios al momento de ofertar y vender los productos que demandan.

\subsection{Planificación de marketing: aspectos organizacionales}

A nivel organizacional, se evidenció que, en las pymes locales, el tema de la planificación de marketing no es un aspecto prioritario en el proceso estratégico por varios factores. Entre ellos, se puede mencionar que únicamente el 36,5\% de empresas indicaron que existe un interés real por la planificación de marketing, mientras que el 32\% de entrevistados manifestaron que toman decisiones basadas en los resultados de una planificación bien estructurada. Esto se debe a que la mercadotecnia no es una prioridad ni se obtienen los recursos necesarios para que los planes se realicen satisfactoriamente; además, no se destina el tiempo requerido a diseñar un plan que sustente las decisiones que se toman por parte de la gerencia.
Otro aspecto relevante consiste en que no existe una investigación formal o bien estructurada sobre los mercados, pues solo el $21,5 \%$ de empresarios señalaron que se investiga el mercado frecuentemente. En este punto, se consideró a los que están totalmente de acuerdo, pues, en algunos casos, se realizan sondeos o encuestas en redes sociales poco serias, que no contribuyen con rigor al proceso de investigación; eso afecta la calidad de la información y los datos como insumo para realizar una planeación estratégica sólida.

Adicionalmente, en las pymes, existe poca participación del personal de ventas en la planificación estratégica, pese a que este se encuentra en permanente contacto con los consumidores, y puede aportar los insumos requeridos para el mejoramiento de los procesos de comercialización y satisfacción de los clientes. Así, se ve afectada la detección de oportunidades del mercado para ser más competitivos y estar mejor posicionados. Asimismo, se corrobora que los factores mencionados (falta de interés, escasos recursos y poca participación de los miembros de las pymes) dificultan la capacidad para aprender y adoptar nuevas técnicas de planificación que sean innovadoras y competitivas. Por ello, se mantienen los resultados negativos: por ejemplo, no se cumple al $100 \%$ con los objetivos planteados, y las pymes no cuentan con herramientas de evaluación y medición de resultados que les permitan controlar eficientemente los recursos invertidos.

\subsection{Mix de marketing}

Las pymes locales aún están enfocadas en la tendencia del marketing 1.0, debido a que el 75,6\% considera que la variable más importante en la que se debe enfocar una estrategia de marketing es el producto (enfoque de calidad). Sin embargo, según las tendencias actuales, las empresas deben trasmitir el interés por hacer del mundo un lugar más ameno para vivir (bienestar 
del cliente), para lo cual deben humanizar las marcas. Esto se debe a que, en múltiples ocasiones, no se logran materializar las características del producto en el mensaje. El más grande y claro ejemplo es el de Coca Cola, que, en la mayor parte de sus spots, busca trasmitir emociones, alegría y unidad, entre otros valores.

En segundo lugar, en el orden de importancia de las variables del mix de marketing, se encuentran el precio y la comunicación. Por ejemplo, debido la idiosincrasia de la sociedad, tiende a haber una inclinación por un precio menor, lo que se explica por la reducción del poder adquisitivo de las familias por la crisis económica que atraviesa el país. Eso conlleva a sacrificar la calidad. En este sentido, es difícil para las pymes caer en una guerra de precios con otras empresas de mayor competencia. Lo correcto sería que el consumidor prefiera una marca por el nivel de satisfacción que esta le genera y no por ser la opción más barata.

La comunicación o forma de llegar a las personas puede ser considerada a nivel local, es decir, de manera muy limitada para lograr colocar y vender productos en otros mercados. Se trata de una variable muy importante, a pesar de que el mix engrana por igual a las cuatro variables. En la actualidad, el mensaje que transmite es insuficiente y poco diferenciado de otras pymes, por lo que es necesario usar otras tendencias más actuales, como content marketing, marketing de influencer y social media marketing, así como otras herramientas relacionadas al mundo digital. Esto se debe a que, a través del internet, se puede viralizar el contenido, promover la calidad del producto y disminuir los costos frente a los medios tradicionales de mercadotecnia.

Por último, la distribución fue la variable a la que las personas encuestadas menos importancia le dieron. Sin embargo, en el contexto de la pandemia, debe ser considerada vital para el funcionamiento creativo de las pymes con el fin de que se fortalezca por medio de estrategias de marketing, dada la necesidad de destinar los recursos a través de la diversificación de los canales de distribución. Esto puede lograrse considerando los problemas como oportunidades: debido al miedo al contagio y a las restricciones del confinamiento, las personas necesitan otras facilidades de compra. Entonces, los especialistas de marketing tienen que multiplicar el interés por innovar. Por ejemplo, hay casos de empresas que han incursionado a nivel local en el e-commerce, pero no todas han sabido aprovechar todos los beneficios de la herramienta. Así, se entiende la importancia de proponer estrategias innovadoras mediante el empleo de herramientas de marketing digital.

\subsection{Importancia de invertir en marketing en las pymes}

Sobre la base de la encuesta realizada, se observa que los representantes de las pymes consideran importante invertir en activos digitales y publicidad online. Se considera esta percepción como el reflejo de la importancia de la digitalización de los negocios. Si bien en otros países ese es un tema bastante común, a nivel local, aún es un campo por explorar, pues las empresas se enfocan únicamente en la producción. Entonces, descuidan las oportunidades que el marketing proporciona, que permiten que las empresas produzcan a partir de la oferta y demanda; es decir, no piensan en la optimización de recursos ni entienden el mercado de la manera más provechosa.

Además, se pudo corroborar que otros empresarios empezaron a considerar importante invertir en nuevos productos y canales de distribución a raíz de la pandemia. Sin embargo, las pymes locales evidenciaron no estar preparadas para afrontar esta nueva realidad 
empresarial, porque, en su mayoría, manejaban canales tradicionales y su producción era constante. Tales circunstancias conllevaron a la falta de innovación. Por ende, gran cantidad de las pymes cerraron y otras aún están tratando de recuperarse económicamente. A partir de lo expuesto, se considera que los canales digitales y la diversificación de productos son variables esenciales en cualquier planificación estratégica, pues es indispensable contar con herramientas novedosas para ser competitivos.

\subsection{Tipos de estrategias y activos digitales en las pymes}

El 44\% de las pymes consultadas manifestó que aplica estrategias digitales y tradicionales; el 39\% indicó que se ha inclinado por las digitales; el $13 \%$ ha optado por las tradicionales; y el $4 \%$ no ha aplicado algún tipo de estrategia de marketing. Es evidente que gran parte de estas organizaciones se han ido adaptando a las exigencias de la globalización, es decir, han digitalizado sus estrategias, incluidas las de mercadotecnia. En el análisis que se está realizando, se considera que el principal activo digital de las pymes corresponde a las redes sociales y las páginas web. Si bien son elementos indispensables en la estrategia digital, requieren de una planificación estructurada que permita mejorar los resultados. Por ello, deben considerarse también algunos elementos tradicionales, como la investigación de mercados, la segmentación, y las estrategias de posicionamiento. Así, se demuestra que el marketing digital es esencial para el desarrollo de las pymes ambateñas. Asimismo, se pudo confirmar que la mayoría de estas organizaciones no cuenta con el personal especializado en marketing. En esos casos, hay un tercero que realiza esa actividad, es decir, administra los activos digitales y diseña las estrategias de marketing, esta situación puede ser considerada como una desventaja, pues, como el encargado no es parte de la empresa, los insumos podrían no reflejar completamente la realidad. Por ello, es importante contar con un encargado propio de mercadotecnia en todas las empresas.

\section{Discusión de resultados}

Considerando los aspectos organizacionales del estudio, fue evidente que, en las pymes locales, la planificación de marketing es deficiente, pues no existe un real interés al respecto. Por ello, no se realizan las inversiones suficientes o se hacen de manera empírica. En concordancia con Pachón (2016), es necesario romper el paradigma de que solo las grandes empresas pueden hacer marketing, pues, en un mercado globalizado, los consumidores se vuelven más exigentes: no solo demandan productos o servicios de calidad, sino que también esperan obtener un valor agregado. Para ello, las empresas deben restructurarse y desarrollar estrategias enfocadas en la gestión de marketing. Entonces, se debe trabajar con el personal de la organización con respecto a la comunicación e información que se trasmite al cliente cuando se le ofrece y brinda un servicio. Esto garantiza y propone confiabilidad y credibilidad tanto para los clientes como para los negocios. A la vez, el marketing que se desarrolle debe ser fresco y no repetitivo, y debe generar información útil y de calidad al cliente para que decida por su compra sin desconfianza.

Por otro lado, Pinargote (2019) señala que la planificación de marketing no asegura el éxito de una empresa; no obstante, disminuye el riesgo de fracaso en un entorno en el que las pymes pueden verse desvalidas ante el alto nivel de competitividad del mercado. A esto se suma lo expuesto por Núñez (2019), que manifiesta que los empresarios deben salir de su zona de confort e invertir en marketing para sus pequeñas organizaciones, pues los beneficios son evidentes: por 
ejemplo, mejor posicionamiento en el mercado, y mayor fidelización y competitividad. Concretamente, la planificación del marketing debe ser proactiva y debe generar nuevas ideas, con el propósito de que los clientes perciban elementos diferenciadores del resto de la competencia, y puedan decidirse de acuerdo a sus expectativas y necesidades primordiales.

Con respecto al mix de marketing, Striedinger (2018) indicó que el marketing digital es el complemento de la mezcla de mercadotecnia tradicional, pues se basa en las cuatro nuevas "ps»: personalisation, participation, peer-to-peer y predictive. Estas estrategias se refieren a la personalización de los productos y servicios; la participación de los consumidores en el marketing mix; la búsqueda de información y recomendaciones; y, como punto más importante, la predicción, es decir, las predicciones de los expertos en marketing sobre el comportamiento de los consumidores a partir de las herramientas tecnológicas para atender mejor sus necesidades y anticiparse a la competencia. De esta manera, el marketing se puede potencializar de una manera personalizada para el tipo de cliente o segmento. Por ello, las organizaciones deben invertir en mejorar su tecnología y en el empleo de la misma en las redes sociales. Además, por medio del marketing en redes, deben buscar lo que quieren los clientes, es decir, deben generar las necesidades del cliente para que este pueda decidirse y llegar al consumo.

Adicionalmente, Kotler, Pfoertsch y Sponholz (2021) manifiestan que, la evolución de las cuatro "ps» del marketing consiste en el marketing $h 2 h$ o human to human. No es un sustituto de las cuatro "ps», sino la manera de adaptar las variables al mundo empresarial actual. El nuevo modelo se puede adaptar a cualquier empresa. Trata de identificar un problema humano con un gran potencial de mercado, para lo cual intervienen tres factores: design thinking (proceso de innovación iterativo), service-dominant logic (creación conjunta de valor en ecosistemas colaborativos) y digitalization (interacción de marketing). De esta manera, el marketing $\mathrm{H} 2 \mathrm{H}$ concuerda con el marketing 4.0, que busca dotar a las marcas de cualidades personales a través de su humanización, criterio con el cual coinciden Toro, Graciano y Gómez (2018).

Con respecto a la inversión en marketing para las pymes, Cafaro (2019) menciona que es vital invertir en inbound marketing para determinar y conocer al consumidor ideal. Asimismo, concluye que los activos digitales son determinantes, pues, en su estudio, determinó que, antes de una compra, el 98\% de consumidores realizan una búsqueda de información sobre el producto o servicio en internet. Asimismo, Zlateva (2021) considera necesario invertir en estrategias de marketing enfocadas en el tema digital, debido a que facilitan un acceso global, son menos costosas que las tradicionales, permiten medir resultados y crear comunidades, y facilitan la personalización de la atención al cliente; además, cada vez es más común que las personas compren en internet, y el big data y la IA son factores claves en la digitalización de los negocios. Cuervo (2021) coincide con Zlateva, pues indica que invertir en IA permite que las empresas innoven y no se vuelvan obsoletas. Con respecto a la IA, Cuervo menciona las siguientes tendencias: big data, chatbot, agentes y asistentes virtuales, blockchain, drones, internet of things (IoT), entre otros.

Frente a lo comentado, Oller y Játiva (2016) consideran que, en Ecuador, las pymes aún no entienden la magnitud de los beneficios que aporta la mercadotecnia y la aplican con cierto grado de empirismo, es decir, no se ejecuta una planificación estratégica bien estructurada. Por ello, se requiere tomar consciencia y fomentar el hábito de planificación de todas sus estrategias. Así mismo, Hernández et al. (2019) indican 
que, en las pymes, las estrategias tradicionales deben ir evolucionado a la par de los avances tecnológicos. En ese sentido, consideran que el marketing digital se ha convertido en el principal aliado en la búsqueda de posicionamiento de este tipo de empresas. Por su parte, Arteaga, Coronel, y Acosta (2018) exponen que el marketing digital ha influenciado en las pymes ecuatorianas, pues, a través de las estrategias implicadas, han podido optimizar su rendimiento, sus procesos y su atención al cliente; también manifestaron que, pese a la resistencia al e-commerce por temor a estafas, es esencial invertir en la digitalización de los negocios, como clave de posicionamiento en el mercado.

Con respecto al tipo de estrategias que se aplican a nivel local, se evidenció que las empresas están realizando estrategias de marketing digital, especialmente en redes sociales. Si bien son fundamentales, también deben apoyarse en otras estrategias para tener una campaña más efectiva. A partir de ello y de la situación que vive el mundo por la pandemia, según Hoyos y Sastoque (2020), las pymes deberían aprovechar estrategias digitales como, por ejemplo, content marketing, social media marketing, páginas web y posicionamiento SEO-SEM, y mailing. A estas ideas se suma lo expuesto por Razak, Maming, Kadir y Sobarsyah (2021), que manifiestan que el social media marketing es la principal tendencia de mercadotecnia que pueden usar las pymes, pues les ayuda a optimizar sus resultados.

Finalmente, la tendencia central del marketing es la de humanizar las marcas. Esto significa darle una personalidad y familiaridad, con la finalidad de conectar con los clientes actuales y potenciales. Para ello, se debe seguir la recomendación de Kotler et al. (2018), que hablan sobre el omnichannel o presencia en varios canales, pues esto incrementa las posibilidades de éxito que puede tener la campańa de marketing digital. Asimismo, en cuanto a las subtendencias, se deben usar aquellas que estén latentes y representen menor costo; de acuerdo con Hubspot \& Talwalker (2020), dichas subtendencias son las redes sociales y los contenidos que permiten producir, como videos o TikTok, viralización de contenidos o memes, marketing de influencers, content marketing, entre otros. De la misma manera, como ya se ha comentado en la investigación, se pueden realizar inversiones en estrategias o activos digitales, como páginas web; plataformas de e-commerce, big data, e IA.

\section{Conclusiones}

Las pymes locales han realizado inversiones en marketing. No obstante, no lo han tenido como un pilar en su planificación estratégica. Por ende, los resultados no han sido los deseados, lo que ha conllevado a que, en ciertos casos, no se invierta más en mercadotecnia. Esto es un gran error, pues, en la actualidad, el marketing digital representa diversas oportunidades para las pymes, ya que les permite acceder a más mercados con un menor presupuesto y con la opción de cuantificar los beneficios obtenidos para, de esta forma, obtener un feedback que facilite la toma de decisiones y permita corregir los errores que afecten su posicionamiento frente a la competencia.

La digitalización de los negocios no es opcional, pues la globalización lleva a que las pymes tengan que adaptarse a los cambios en el mercado. Para ello, tienen que invertir en función de los resultados que obtengan en social media marketing, content marketing y en todas las estrategias relacionadas a las redes sociales, dado que contribuyen al incremento de las ventas y al desarrollo propio de las organizaciones. Entonces, el tema digital es imprescindible para la aplicación de las estrategias off y online, por su facilidad de adecuarse a las exigencias del mercado y a la capacidad de cada una de las organizaciones.

Contabilidad y Negocios (16) 32, 2021 / ISSN 1992-1896 


\section{Referencias}

Alarcón, C., \& Granda, M. (2018). El marketing y la fidelización empresarial como apuesta estratégica para pymes en Ecuador. Domio de las Ciencias, 4(1), 131140. https://doi.org/10.23857/dc.v4i1.727

Alin, L. (2019). The impact of industrial revolution to marketing. Electronic Research Journal of Social Sciences and Humanities, 1(4), 114-122. Recuperado de https:// www.researchgate.net/publication/336666822 [Consulta: 26 de abril del 2021].

Andhyka, B. (2020). Marketing 4.0 a Literature Review. IOSR Journal of Business and Management (IOSR$J B M), 22(4)$, 49-52. Recuperado de https://www. academia.edu/43015116/Marketing_4_0_a_Literature_Review [Consulta: 26 de abril del 2021].

Arteaga, J., Coronel, V., \& Acosta, M. (2018). Influencia del marketing digital en el desarrollo de las MIPYME's en Ecuador. Revista Espacios, 39(47). Recuperado de http://www.revistaespacios.com/a18v39n47/ a18v39n47p01.pdf [Consulta: 26 de abril del 2021].

Cafaro, R. (2019). El comprador ideal, una estrategia de marketing para las pymes modernas. Business Innova Sciences, 1(1) 70-79. Recuperado de https://innovasciencesbusiness.org/index.php/ISB/article/view/5 [Consulta: 23 de diciembre del 2020].

Camacho, L. (2019). Marketing digital en las pymes. Documentos de Trabajo ECACEN, 2, 1-10. https://doi. org/10.22490/ECACEN.3489

Cuervo, C. (2021). Efectos de la inteligencia artificial en las estrategias de marketing: Revisión de literatura. Revista Internacional de Investigación en Comunicación aDResearch ESIC, 24(24), 26-41. https://doi.org/10.7263/ adresic-024-02

Enríquez, C. (2019, 9 de diciembre). 10 tendencias en marketing para 2020. Forbes México. Recuperado de https://www.forbes.com.mx/10-tendencias-enmarketing-para-2020/ [Consulta: 22 de diciembre del 2020].
Erragcha, N., \& Romdhane, R. (2014). New faces of marketing in the era of the web: From marketing 1.0 to marketing 3.0. TechMind Research, Society, 2(2), 137142. https://doi.org/10.17722/jorm.v2i2.46

Fuciu, M., \& Dumitrescu, L. (2018). From marketing 1.0 to marketing 4.0 - the evolution of the marketing concept in the context of the 21 st century. International Conference Knowledge-Based Organization, 24(2), 43-48. https://doi.org/10.1515/kbo-2018-0064

Gonzáles, A. (2017). Nuevas tendencias del marketing en las ciencias económicas y administrativas. Cuadernos Latinoamericanos de Administración, 13(25), 5-6. https://doi.org/10.18270/cuaderlam.v13i25.2384

Goyzueta, S. (2015). Big data marketing: una aproximación. Revista Perspectivas, 18(35), 147-158. Recuperado de http://www.scielo.org.bo/pdf/rp/n35/n36_a07. pdf [Consulta: 5 de abril del 2021].

Hernández Sampieri, R., Fernández, C., \& Baptista, P. (2014). Metodología de la investigación. México, D. F.: McGraw-Hill. Recuperado de: https:/www.uca. ac.cr/wp-content/uploads/2017/10/Investigacion.pdf [Consulta: 3 de abril del 2020].

Hernández, L., López, D., \& Salcedo, O. (2019). Diseño del proceso de marketing para estructurar un plan de negocio interno dirigido a pymes en Colombia. Espacios, 40(13), 3-13. Recuperado de https:/www. revistaespacios.com/a19v40n13/a19v40n13p03.pdf [Consulta: 23 de diciembre del 2020].

Hoyos, S., \& Sastoque, J. (2020). Marketing digital como oportunidad de digitalización de las pymes en Colombia en tiempo del Covid-19. Revista Cientifica Anfibios, 3(1), 39-46. https://doi.org/10.37979/ afb.2020v3n 1.60

Hubspot \& Talwalker. (2020). Tendencias de las redes sociales. Un informe detallado para impulsar tu estrategia de redes sociales. Massachusetts: Hubspot \& Talwalker.

Instituto Nacional de Estadísticas y Censos (INEC). (2019). Directorio de empresas y establecimientos. Quito: INEC. 
Recuperado de https://www.ecuadorencifras.gob.ec/ directoriodeempresas/ [Consulta: 3 de abril del 2020].

Kotler, P., Kartajaya, H., \& Setiawan, I. (2018). Marketing 4.0: Transforma tu estrategia para atraer al consumidor digital. Madrid: LID Editorial.

Kotler, P., Pfoertsch, W., \& Sponholz, U. (2021). The new paradigm: $\mathrm{H} 2 \mathrm{H}$ marketing. En $\mathrm{H} 2 \mathrm{H}$ Marketing (pp. 29-90). Cham: Springer. https://doi. org/10.1007/978-3-030-59531-9_2

León, J., Garzón, A., Meza, C., \& Macias, J. (2018). E-commerce: método de crecimiento económico para las pymes (Trabajo de grado, Facultad de Ingeniería y Ciencias Básicas, Institución Universitaria Politécnico Grancolombiano. Bogotá, Colombia). Recuperado de https:// alejandria.poligran.edu.co/handle/10823/1219 [Consulta: 5 de abril del 2021].

Lovato, S., López, M., \& Acosta, M. (2018). Incidencia de las herramientas administrativas y el marketing en el desarrollo microempresarial de la provincia de Santa Elena de Ecuador. Espacios, 39(24), 21-26. Recuperado de https://www.revistaespacios.com/a18v39n24/ a18v39n24p21.pdf [Consulta: 4 de abril del 2021].

Mendivelso, H., \& Lobos, F. (2019). La evolución del marketing: una aproximación integral. Revista Chilena de Economía y Sociedad, 13(1), 59-70. Recuperado de https://rches.utem.cl/articulos/la-evolucion-del-marketing-una-aproximacion-integral/ [Consulta: 24 de abril del 2021].

Mieles , J., Albán, A., Valdospin, J., \& Vera, D. (2018). E-commerce:un factor fundamental para el desarrollo empresarial en el Ecuador. Revista Cientifica ECOCIENCIA, 1-17. Recuperado de https://search.proquest. com/openview/799c6f7ee6e3cd5e0dbe85e40cf68f40/ 1.pq-origsite $=$ gscholar $\& \mathrm{cbl}=2043236 \#:-:$ text $=\mathrm{E} \% 2$ Dcommerce $\% 20$ fuente $\% 20$ principal $\% 20$ que,que $\%$ 20sus\%20 costos\%20sean\%20menores [Consulta: 24 de abril del 2021].

Núñez, B. (2019). Importancia de las nuevas tendencias de marketing en las pymes. Revista de Investigación
Sigma, 6(2), 62-68. https://doi.org/10.24133/sigma. v6i2.1674

Olivier, E. (2020, 30 de julio). 5 tendencias de inbound marketing en este 2020. Forbes México. Recuperado de https://www.forbes.com.mx/5-tendencias-de-inboundmarketing-en-este-2020/ [Consulta: 5 de abril del 2021].

Oller, M., \& Játiva, E. (2016). La competitividad en las pequeñas y medianas empresas (pyme). En M. Oller et al. (Eds.), Tendencias y retos del marketing en Ecuador 2015 (pp. 22-42). Granada: Macasar Ediciones. Recuperado de http://www.revistalatinacs.org/16SLCS/ Martin1.pdf [Consulta: 3 de abril del 2020].

Ortegón, L., \& Gómez, A. ( 2016). Gestión del marketing sensorial sobre la experiencia del consumidor. Revista de Ciencias Sociales, 22(3), 67-83. https://doi. org/10.31876/rcs.v22i3.24869

Pachón, M. (2016). El marketing en las pymes. CITAS, 2(1), 49-55. https://doi.org/10.15332/24224529.5179

Pažèraitè, A., \& Repovienè, R. (2018). Content Marketing Decisions for Effective Internal Communication. Sciendo, 79(1), 117-130. https://doi.org/10.1515/ mosr-2018-0008

Pérez, S. (2020). El marketing 4.0 y su influencia en los consumidores. Revista Neuronum, 6(2), 173-179. Recuperado de http://eduneuro.com/revista/index. $\mathrm{php} /$ revistaneuronum/article/view/230 [Consulta: 24 de abril del 2021].

Pinargote, K. (2019). Importancia del marketing en las empresas. FIPCAEC, 4(10), 77-96. Recuperado de https://fipcaec.com/index.php/fipcaec/article/view/38 [Consulta: 2 de enero del 2021].

Razak, A., Maming, J., Kadir, N., \& Sobarsyah, M. (2021). Brand resonancing capability: the mediating role between social media marketing and SMEs marketing performance. Academy of Entrepreneurship Journal, 27(1). Recuperado de https://www.abacademies.org/ articles/brand-resonancing-capability-the-mediating-

Contabilidad y Negocios (16) 32, 2021 / ISSN 1992-1896 
role-between-social-media-marketing-and-smes-marketing-performance-10098.html [Consulta: 24 de abril del 2021].

Real, I., Leyva, A., \& Heredia, J. (2014). Uso e impacto de las redes sociales en las estrategias de marketing de las pymes. Revista de Investigación Académica sin Frontera, 7(19), 1-24. https://doi.org/10.46589/rdiasf.v0i19.47

Rivera, M. (2015). La evolución de las estrategias de marketing en el entorno digital: implicaciones juridicas (Tesis de doctorado, Departamento de Derecho Privado, Universidad Carlos III de Madrid. Madrid, España). Recuperado de https://e-archivo.uc3m.es/bitstream/ handle/10016/22498/rosario_rivera_tesis.pdf [Consulta: 11 de febrero del 2021].

Rodriguez, C., Camargo, P., González, J., \& Gorrín, J. (2015). Ventajas y desventajas del marketing digital. Revista Convicciones, 2(3), 24-31. Recuperado de https://www. fesc.edu.co/Revistas/OJS/index.php/convicciones/ article/view/209 [Consulta: 2 de enero del 2021].

Rodríguez, M., Pineda, D., \& Castro, C. (2020). Tendencias del marketing moderno, una revisión teórica. Revista Espacios, 41(27), 306-322. Recuperado de https:// www.revistaespacios.com/a20v41n27/a20v41n27p26. pdf [Consulta: 18 de noviembre del 2020].

Ruizalba, J., Vallespín, M., \& Pérez, J. (2015). Gestión del conocimiento y orientación al marketing interno en el desarrollo de ventajas competitivas en el sector hotelero. Investigaciones Europeas de Dirección y Economía de la Empresa (IEDEE), 21(2), 84-92. https://doi. org/10.1016/j.iedee.2014.03.001

Saboga, L., \& Rojas, S. (2020). Evolución del marketing experiencial: una aproximación teórica a su definición. Revisión Sistemática de Literatura, 16(1), 94-107. https://doi.org/10.18041/1900-3803/entramado. 1.6140

Striedinger, M. (2018). El marketing digital transforma la gestión de pymes en Colombia. Cuadernos Latino- americanos de Administración, 14(27). https://doi.org/ 10.18270/cuaderlam.v14i27.2652

Suárez, T. (2018). Evolución del marketing 1.0 al 4.0. Redmarka, Revista de Marketing Aplicado, 01(22), 209-227. https://doi.org/10.17979/redma.2018.01.022.4943

Taylor, B. (2019, 23 de diciembre). 10 tendencias esenciales de marketing para 2020. Launch Metrics. Recuperado de https://www.launchmetrics.com/es/recursos/blog/ tendencias-marketing-2020 [Consulta: 2 de enero del 2021].

Toro, M., Graciano, V., \& Gómez, M. (2018). Humanización de marca Coca-Cola. Revista CIES, 9(2), 156-184. Recuperado de http://www.escolme.edu.co/revista/ index.php/cies/article/view/206 [Consulta: 4 de abril del 2021].

Viteri, F., Herrera, L., \& Bazurto, A. (2017). Las tendencias del marketing: Cuáles son y definiciones. Revista Cientifica Mundo de la Investigación y el Conocimiento, 1(5), 974-988. https://doi.org/10.26820/recimundo/1.5.2017.974-988

Warrink, D. (2015). The marketing mix in a marketing 3.0 context. University of Twente, 4(4), 7-30.https://doi. org/10.18775/ijied.1849-7551-7020.2015.44.2001

Wereda, W., \& Woźniak, J. (2019). Building relationships with customer 4.0 in the era of marketing 4.0: The case study of innovative enterprises in Poland. Social Science, 8(177). https://doi.org/10.3390/socsci8060177

Zlateva, D. (2021). Big data world and the new marketing horizons. Revista Inclusiones, 8, 19-535. Recuperado de https://revistainclusiones.org/index.php/inclu/article/view/2558 [Consulta: 22 de abril del 2021 ].

Fecha de recepción: 02 de mayo de 2021 Fecha de aceptación: 09 de junio de 2021 Correspondencia: juan.c.suarez.p@pucesa.edu.ec operez@pucesa.edu.ec 